\title{
NONLINEAR STATE FEEDBACK CONTROL OF SECOND-ORDER NONMINIMUM-PHASE NONLINEAR SYSTEMS
}

\author{
C. Kravaris and P. Daoutidis \\ Department of Chemical Engineering, The University of Michigan, Ann Arbor, MI 48109, U.S.A.
}

(Received 17 October 1989; received for publication 28 November 1989)

\begin{abstract}
The present work addresses the problem of synthesizing nonlinear state feedback controllers for second-order nonminimum-phase nonlinear systems. The concept of a first-order nonlinear all-pass is first introduced. A class of static state feedback control laws is then developed that makes the closed-loop system equivalent, under an appropriate coordinate transformation, to a nonlinear first-order all-pass in series with a linear first-order lag. A particular control law from this class is calculated that results in ISE-optimal response. The performance of the proposed methodology in set point tracking is evaluated through numerical simulations in a CSTR example.
\end{abstract}

\section{INTRODUCTION}

Major contributions in the area of linear process control in the last decade have established the idea that a controller must explicitly or implicitly generate a process inverse (Garcia and Morari, 1982). When dealing with minimum-phase linear systems, such an inverse is stable and can be used for controller synthesis. When dealing with nonminimum-phase linear systems, an appropriate decomposition of the process model into a part with stable inverse and a part with unstable inverse is necessary and the controller must invert only the part with stable inverse. In the context of linear state feedback, the same idea arises in placing closed-loop poles at the left-half plane zeros and at the reflection of the right-half plane zeros. Such a control strategy has been shown to be ISE-optimal for step changes.

In the field of nonlinear process control, the idea of a controller that generates a process inverse is central in general synthesis methods for minimum-phase systems, like the nonlinear IMC structure (Economou et al., 1986; Parrish and Brosilow, 1988), which explicitly generates a process inverse on-line and the input/ output linearization method (Kravaris and Chung, 1987), which implicitly generates a process inverse. The latter has been shown to lead to ISE-optimal responses for step changes (Kravaris, 1988). However, the control of nonminimum-phase nonlinear systems in this vein remains a major unmet challenge.

In this work, a control law for second-order nonminimum-phase nonlinear systems will be developed, that leads to an ISE-optimal closed-loop response for changes in the set point. The proposed methodology hopes to serve as a starting point for the development of a more general framework for the control of nonminimum-phase nonlinear systems and to motivate further research effort in this area.
In Section 2 the characterization of nonminimumphase behavior for second-order systems will be reviewed following the approach of Byrnes and Isidori (1985). In Section 3, a nonlinear analog of the linear first-order all-pass will be introduced. Section 4 will review a standard result for $I S E$-optimal state feedback control of linear second-order nonminimum-phase systems and will motivate the development that follows. In Section 5, a class of control laws will be developed that lead to a closed-loop response of a nonlinear first-order all-pass in series with a linear first-order lag. In Section 6, a particular control law from this class will be calculated, that results in $I S E$-optimal closed-loop response in the limit as the time constant of the linear lag tends to zero. Finally, Section 7 will illustrate the application of the proposed control methodology and evaluate its performance in a CSTR example.

\section{CHARACTERIZATION OF MINIMUM-PHASE AND NONMINIMUM-PHASE BEHAVIOR IN SECOND-ORDER SYSTEMS}

\subsection{Linear systems}

Nonminimum-phase behavior is well-known and well-characterized in linear systems. It arises from the presence of right-half-plane zeros in the open-loop transfer function, which contribute additional phase lag without changing the gain of the system. In what follows, we provide an alternative characterization of the nonminimum-phase behavior of linear systems, that will allow generalization to nonlinear systems.

Consider a second-order linear system of the form:

$$
\begin{aligned}
& \dot{x}_{1}=a_{11} x_{1}+a_{12} x_{2}+b_{1} u, \\
& \dot{x}_{2}=a_{21} x_{1}+a_{22} x_{2}+b_{2} u, \\
& y=c_{1} x_{1}+c_{2} x_{2},
\end{aligned}
$$


with

$$
c_{1} b_{1}+c_{2} b_{2} \neq 0 \text {. }
$$

This system has transfer function:

$\frac{\left(c_{1} b_{1}+c_{2} b_{2}\right) s-\left(a_{22} c_{1} b_{1}+a_{11} c_{2} b_{2}-a_{12} c_{1} b_{2}-a_{21} c_{2} b_{1}\right)}{s^{2}-\left(a_{11}+a_{22}\right) s+\left(a_{11} a_{22}-a_{12} a_{21}\right)}$

and consequently a zero at:

$$
z=\frac{a_{22} c_{1} b_{1}+a_{11} c_{2} b_{2}-a_{12} c_{1} b_{2}-a_{21} c_{2} b_{1}}{c_{1} b_{1}+c_{2} b_{2}}
$$

Based on the above transfer function description, the linear system (1) will be minimum-phase if $z<0$ and nonminimum-phase if $z>0$. An alternative characterization of (non)minimum-phase behavior can be obtained by transforming (1) into its output controllability canonical form. In particular, through the coordinate change:

$$
\left[\begin{array}{l}
\zeta_{1} \\
\zeta_{2}
\end{array}\right]=\left[\begin{array}{cc}
\kappa b_{2} & -\kappa b_{1} \\
c_{1} & c_{2}
\end{array}\right]\left[\begin{array}{l}
x_{1} \\
x_{2}
\end{array}\right],
$$

where $\kappa$ is an arbitrary nonzero constant, (1) is transformed into:

$$
\begin{aligned}
& \dot{\zeta}_{1}=\tilde{a}_{11} \zeta_{1}+\tilde{a}_{12} \zeta_{2}, \\
& \dot{\zeta}_{2}=\tilde{a}_{21} \zeta_{1}+\tilde{a}_{22} \zeta_{2}+\tilde{b}_{2} u, \\
& y=\zeta_{2},
\end{aligned}
$$

where

$$
\begin{aligned}
& \tilde{a}_{11}=\frac{\left(a_{22} b_{1}-a_{12} b_{2}\right) c_{1}+\left(a_{11} b_{2}-a_{21} b_{1}\right) c_{2}}{c_{1} b_{1}+c_{2} b_{2}} \\
& \tilde{a}_{12}=\kappa \frac{\left(a_{11} b_{1}+a_{12} b_{2}\right) b_{2}-\left(a_{21} b_{1}+a_{22} b_{2}\right) b_{1}}{c_{1} b_{1}+c_{2} b_{2}} \\
& \tilde{a}_{21}=\frac{1}{\kappa} \frac{\left(a_{11} c_{2}-a_{12} c_{1}\right) c_{1}+\left(a_{21} c_{2}-a_{22} c_{1}\right) c_{2}}{\left(c_{1} b_{1}+c_{2} b_{2}\right)} \\
& \tilde{a}_{22}=\frac{\left(a_{12} c_{1}+a_{22} c_{2}\right) b_{2}-\left(a_{11} c_{1}+a_{21} c_{2}\right) b_{1}}{c_{1} b_{1}+c_{2} b_{2}} \\
& \tilde{b}_{2}=c_{1} b_{1}+c_{2} b_{2} .
\end{aligned}
$$

The internal structure of the output controllability canonical form has the following very important properties:

- The input affects only the second state equation.

- The output is equal to the second state.

As a result of this internal structure, it can be easily seen from (6) that $\tilde{a}_{11}$ is equal to the system zero. Furthermore, the stability of the first state equation of (5), with $\zeta_{2}=y$ assuming a fixed value, is determined by the sign of $a_{11}$ (i.e. the system zero) and thus it can be used as an alternative way of characterizing the system as minimum or nonminimum-phase. In particular:

Proposition $I$-The second-order linear system (1) or (5) is minimum-phase if the dynamic system:

$$
\zeta_{1}=\tilde{a}_{11} \zeta_{1}+\tilde{a}_{12} y,
$$

is stable, where $y$ assumes a fixed constant value. It is nonminimum-phase if (7) is unstable.
Remark 1 -A straightforward calculation of the system inverse on the basis of (5) shows that (7) is exactly the dynamics of the inverse.

\subsection{Nonlinear systems}

In nonlinear systems, a transfer function description and therefore an explicit zero characterization is not possible. However, there is a normal form for nonlinear systems that has analogous properties with the output controllability canonical form for linear systems and leads to the definition of zero dynamics, a concept analogous to the zeros for linear systems. The normal form as well as the concept of zero dynamics were developed by Byrnes and Isidori (1985) for SISO systems of arbitrary order and then generalized to MIMO systems by Isidori and Moog (1988). Using these concepts, one can obtain an explicit characterization of the (non)minimum-phase behavior in nonlinear systems of arbitrary order. In what follows, we review the case of second-order systems.

Consider a second-order nonlinear system of the form:

$$
\begin{aligned}
\dot{x}_{1} & =f_{1}\left(x_{1}, x_{2}\right)+g_{1}\left(x_{1}, x_{2}\right) u, \\
\dot{x}_{2} & =f_{2}\left(x_{1}, x_{2}\right)+g_{2}\left(x_{1}, x_{2}\right) u, \\
y & =h\left(x_{1}, x_{2}\right),
\end{aligned}
$$

where

$$
g_{1}\left(x_{1}, x_{2}\right) \frac{\partial h}{\partial x_{1}}\left(x_{1}, x_{2}\right)+g_{2}\left(x_{1}, x_{2}\right) \frac{\partial h}{\partial x_{2}}\left(x_{1}, x_{2}\right) \neq 0
$$

and denote by $t\left(x_{1}, x_{2}\right)$ a nontrivial particular solution of the partial differential equation:

$$
g_{1}\left(x_{1}, x_{2}\right) \frac{\partial t}{\partial x_{1}}+g_{2}\left(x_{1}, x_{2}\right) \frac{\partial t}{\partial x_{2}}=0
$$

Then the change of coordinates:

$$
\begin{aligned}
& \zeta_{1}=\gamma\left[t\left(x_{1}, x_{2}\right)\right], \\
& \zeta_{2}=h\left(x_{1}, x_{2}\right),
\end{aligned}
$$

where $\gamma$ is an arbitrary nonconstant function, transforms (8) into a system of the form:

$$
\begin{aligned}
& \zeta_{1}=F_{1}\left(\zeta_{1}, \zeta_{2}\right), \\
& \zeta_{2}=F_{2}\left(\zeta_{1}, \zeta_{2}\right)+G_{2}\left(\zeta_{1}, \zeta_{2}\right) u, \\
& y=\zeta_{2}
\end{aligned}
$$

where

$$
\begin{aligned}
& F_{1}\left(\zeta_{1}, \zeta_{2}\right)=\gamma^{\prime}(t)\left(\frac{\partial t}{\partial x_{1}} f_{1}+\frac{\partial t}{\partial x_{2}} f_{2}\right), \\
& F_{2}\left(\zeta_{1}, \zeta_{2}\right)=\frac{\partial h}{\partial x_{1}} f_{1}+\frac{\partial h}{\partial x_{2}} f_{2}, \\
& G_{2}\left(\zeta_{1}, \zeta_{2}\right)=\frac{\partial h}{\partial x_{1}} g_{1}+\frac{\partial h}{\partial x_{2}} g_{2},
\end{aligned}
$$

with the understanding that the right-hand sides of (13) are transformed into the $\left(\zeta_{1}, \zeta_{2}\right)$ coordinate system. 
The realization (12) is called the Byrnes-Isidori normal form and is the nonlinear analog of the output controllability canonical form (5) of linear systems. It is characterized by the same properties as (5):

- The input affects only the second state equation.

- The output is equal to the second state.

Definition 1 -The zero dynamics of the secondorder nonlinear system (8) or (12) is the dynamic system:

$$
\dot{z}=F_{1}(z, y),
$$

where $y$ assumes a fixed constant value.

The zero dynamics (14) is exactly the dynamics of the inverse of the nonlinear system if calculated on the basis of (12), or equivalently, the internal dynamics of the system when the output is constrained to remain constant for all times (in analogy with the block transmission property of the zeros in linear systems).

Remark 2-If Definition 1 provides a meaningful concept of nonlinear zeros, then it is reasonable to expect that (14) must generate the entire family of zeros of the linear approximation of (12), as the steady state moves in state space. Indeed, the pole of the linear approximation of (14) around the steady state $\left(z_{s}, y_{s}\right)$ is $\partial F_{1} / \partial z\left(z_{s}, y_{s}\right)$, which is equal to the zero of the linear approximation of (12) around the steady-state $\left(\boldsymbol{\zeta}_{1 s}, \zeta_{2 s}\right)=\left(z_{s}, \boldsymbol{y}_{s}\right)$.

In analogy with Proposition 1, we can give the following definition of (non)minimum-phase behavior for nonlinear systems:

Definition 2 - The second-order nonlinear system (8) or (12) is said to be minimum-phase if its zero dynamics (14) is locally asymptotically stable at each steady state. It is said to be nonminimum-phase if the zero dynamics (14) is unstable.

For the second-order nonlinear system of interest, a characterization of (non)minimum-phase behavior is obtained by the following proposition:

Proposition 2-A second-order nonlinear system (8) or (12) is minimum-phase if and only if:

$$
\begin{gathered}
\frac{\partial F_{1}}{\partial z}(z, y)<0 \text { in a neighborhood of the line } \\
F_{1}(z, y)=0 .
\end{gathered}
$$

It is nonminimum-phase if and only if:

$$
\begin{gathered}
\frac{\partial F_{1}}{\partial z}(z, y)>0 \text { in a neighborhood of the line } \\
F_{1}(z, y)=0 .
\end{gathered}
$$

The proof of the above proposition is a standard application of Lyapunov's direct method. For every fixed $y$, the function $V(z)=\left[F_{1}(z, y)\right]^{2}$ is an appropriate Lyapunov function for the application of the method.

Remark 3-There may be cases where a nonlinear system is minimum-phase for part of the steady state line $F_{1}(z, y)=0$ and nonminimum-phase for the rest.

\section{FIRST-ORDER ALL-PASS}

The purpose of this section is to re-examine the properties of the (linear) first-order all-pass by studying its state-space realizations and introduce for the first time a concept of a nonlinear first-order all-pass. This concept will play a crucial role in the development of nonlinear state feedback laws for nonminimum-phase second-order systems in the subsequent sections.

\subsection{Linear case}

Recall from classical control theory that a linear dynamic system with transfer function:

$$
\frac{1-T s}{1+T s},
$$

where $T>0$ is called a first-order all-pass. Apart from its well-known frequency response characteristics, a first-order all-pass can be completely characterized by the following properties:

1. It is stable.

2. Its zero is at the "mirror image" of its pole with respect to the imaginary axis.

3. It has static gain equal to 1 .

One can use the above three properties to obtain an alternative characterization of an all-pass in state space. Starting with a general first-order system of the form:

$$
\begin{aligned}
& \dot{x}=\alpha x+\beta u, \\
& y=\gamma x+\delta u,
\end{aligned}
$$

with

$$
\beta \neq 0
$$

and imposing Properties 1-3, one can casily deduce that the following conditions must be satisfied:

1. $\alpha<0$,

2. $\alpha-\beta \frac{\gamma}{\delta}=-\alpha$,

3. $\delta-\beta \frac{\gamma}{\alpha}=1$,

which are equivalent to:

1. $\alpha<0$,

$2^{\prime} \cdot \gamma=-2 \frac{\alpha}{\beta}$,

$3^{\prime} . \delta=-1$. 
Consequently, the general state-space representation of a first-order all-pass is:

$$
\begin{aligned}
& \dot{x}=\alpha x+\beta u, \\
& y=-2 \frac{\alpha}{\beta} x-u,
\end{aligned}
$$

where $\alpha<0$ and $\beta \neq 0$, and this has transfer function:

$$
\frac{1+\frac{1}{\alpha} s}{1-\frac{1}{\alpha} s}
$$

\subsection{Nonlinear case}

In this section we will introduce the concept of a nonlinear first-order all-pass, in analogy with the state-space realization properties of a linear first-order all-pass discussed previously. Consider a general first-order nonlinear dynamic system of the form:

$$
\begin{aligned}
& \dot{x}=\phi(x, u), \\
& y=w(x, u),
\end{aligned}
$$

where

$$
\frac{\partial \phi}{\partial u}(x, u) \neq 0 .
$$

Since Property 2 of the previous subsection refers to the system zero, we need to determine the zero dynamics of (16). Similarly to the case of the nonlinear second-order system discussed in the previous section, the zero dynamics of (16) is the dynamics of its inverse or its internal dynamics when the output assumes a fixed constant value, i.e. it is the dynamic system:

$$
\dot{x}=\tilde{\phi}(x, y),
$$

where $\tilde{\phi}(x, y)=\phi[x, \psi(x, y)]$ and where $u=\psi(x, y)$ is the implicit function defined as the solution of $y=w(x, u)$. The stability properties of (17), i.e. the sign of:

$$
\frac{\partial \tilde{\phi}}{\partial x}=\frac{\partial \phi}{\partial x}+\frac{\partial \phi \partial \psi}{\partial u \partial x}=\begin{aligned}
& \partial \phi \\
& \partial x
\end{aligned} \quad \frac{\partial \phi}{\partial u} \frac{\frac{\partial w}{\partial x}}{\frac{\partial w}{\partial u}}
$$

will determine whether $(16)$ is minimum-phase or not.

Remark 4-If we consider the linear approximation of (16) around the steady state $\left(u_{s}, x_{s}, y_{s}\right)$, this has a pole at:

$$
\frac{\partial \phi}{\partial x}\left(x_{s}, u_{s}\right)
$$

and a zero at

$$
\frac{\partial \tilde{\phi}}{\partial x}\left(x_{s}, y_{s}\right)=\frac{\partial \phi}{\partial x}\left(x_{s}, u_{s}\right)-\frac{\partial \phi}{\partial u}\left(x_{s}, u_{s}\right) \frac{\frac{\partial w}{\partial x}\left(x_{s}, u_{s}\right)}{\frac{\partial w}{\partial u}\left(x_{s}, u_{s}\right)}
$$

Thus, we see that the family of poles of the zero dynamics (17) generates the entire family of zeros of
(16), as $\left(u_{s}, x_{s}, y_{s}\right)$ runs through all possible system steady states.

Definition 3-A first-order nonlinear dynamic systern of the form (16) will be called a first-order all-pass, if the following conditions are satisfied:

1. $\frac{\partial \phi}{\partial x}(x, u)<0$,

2. $\frac{\partial \phi}{\partial x}(x, u)-\frac{\partial \phi}{\partial u}(x, u) \frac{\frac{\partial w}{\partial x}(x, u)}{\frac{\partial w}{\partial u}(x, u)}=-\frac{\partial \phi}{\partial x}(x, u)$,

3. $w(x, u)=u$,

for all $x, u$ on the line $\phi(x, u)=0$.

Condition 1 expresses the stability requirement for the dynamic system (16).

Condition 2 is a nonlinear analog of the requirement that "the zero is at the mirror image of the pole with respect to the imaginary axis".

Finally, Condition 3 states that the static gain of the system is equal to 1 .

Proposition 3-A first-order nonlinear dynamic system of the form (16) will be a first-order all-pass if and only if the following conditions are satisfied:

$$
\begin{aligned}
& \text { 1. } \frac{\partial \phi}{\partial x}(x, u)<0, \\
& 2^{\prime} \cdot \frac{\partial w}{\partial x}(x, u)=-2 \frac{\frac{\partial \phi}{\partial x}(x, u)}{\frac{\partial \phi}{\partial u}(x, u)}, \\
& \text { 3'. } \frac{\partial w}{\partial u}(x, u)=-1,
\end{aligned}
$$

for all $x, u$ on the line $\phi(x, u)=0$.

Conditions $2^{\prime}$ and $3^{\prime}$ result from Conditions 2 and 3 of Definition 3, by a straighforward application of the implicit function theorem.

Remark 5-Notice that for a given $\phi$, Conditions $2^{\prime}$ and $3^{\prime}$ of Proposition 3 do not uniquely determine $w$.

Proposition 4-A nonlinear first-order all-pass of the form (16) has the following properties:

- Its definition is coordinate-independent.

- Its linear approximation around the steady-state $\left(x_{s}, u_{s}\right)$ has transfer function:

$$
\frac{\frac{\partial \phi}{\partial x}\left(x_{s}, u_{s}\right)+s}{\frac{\partial \phi}{\partial x}\left(x_{s}, u_{s}\right)-s}
$$

Proof:

- Under the coordinate change $x=\Xi(\xi)$, (16) becomes:

$$
\begin{aligned}
& \xi=\hat{\phi}(\xi, u), \\
& y=\hat{w}(\xi, u),
\end{aligned}
$$


where $\bar{\phi}(\xi, u)=\phi(\Xi(\xi), u) / \Xi^{\prime}(\xi)$ and $\hat{w}(\xi, u)=$ $w(\Xi(\xi), u)$. It is easy to see that if $\phi$ and $w$ satisfy Conditions $1,2^{\prime}$ and $3^{\prime}$, then $\hat{\phi}$ and $\hat{w}$ will also satisfy them.

- Consider the linear approximation of (16) around the steady state $\left(x_{s}, u_{s}\right)$. This has transfer function i.e. the closed-loop response is the one of a linear first-order all-pass in series with a linear firstorder lag. In the limit as $\epsilon \rightarrow 0$, the closed-loop response is $I S E$-optimal for step changes in the set point $v$.

$$
\frac{\left[\frac{\partial w}{\partial u}\left(x_{s}, u_{s}\right)\right] s+\left[\frac{\partial \phi}{\partial u}\left(x_{s}, u_{s}\right) \frac{\partial w}{\partial x}\left(x_{s}, u_{s}\right)-\frac{\partial \phi}{\partial x}\left(x_{s}, u_{s}\right) \frac{\partial w}{\partial u}\left(x_{s}, u_{s}\right)\right]}{s-\frac{\partial \phi}{\partial x}\left(x_{s}, u_{s}\right)} .
$$

Substituting the expressions for the partial derivatives of $w,(22)$ and (23), we immediately obtain (24).

\section{ISE-OPTIMAL STATE FEEDBACK CONTROL OF SECOND-ORDER NONMINIMUM-PHASE \\ LINEAR SYSTEMS}

Consider a second-order linear system of the form (1), where:

$$
c_{1} b_{1}+c_{2} b_{2} \neq 0
$$

and

$$
\left[\left(a_{11} b_{1}+a_{12} b_{2}\right) b_{2}-\left(a_{21} b_{1}+a_{22} b_{2}\right) b_{1}\right] \neq 0
$$

and assume that its zero is positive. Then, under the state feedback control law:

$$
u=\lambda v-k_{1} x_{1}-k_{2} x_{2}
$$

where

$$
\begin{aligned}
& \lambda=\frac{-\frac{1}{\epsilon}}{\left(c_{1} b_{1}+c_{2} b_{2}\right)} \neq 0, \\
& k_{1}=\frac{\left(a_{11} b_{2}-a_{21} b_{1}\right)\left[\left(a_{11}+\frac{1}{\epsilon}\right)\left(2 c_{2} b_{2}+c_{1} b_{1}\right)-a_{21} c_{2} b_{1}\right]+\left(a_{22} b_{1}-a_{12} b_{2}\right)\left[\left(a_{11}+\frac{1}{\epsilon}\right) c_{1} b_{2}-a_{21}\left(2 c_{1} b_{1}+c_{2} b_{2}\right)\right]}{\left[\left(a_{11} b_{1}+a_{12} b_{2}\right) b_{2}-\left(a_{21} b_{1}+a_{22} b_{2}\right) b_{1}\right]\left(c_{1} b_{1}+c_{2} b_{2}\right),} \\
& k_{2}=\frac{\left(a_{11} b_{2}-a_{21} b_{1}\right)\left[a_{12}\left(2 c_{2} b_{2}+c_{1} b_{1}\right)-\left(a_{22}+\frac{1}{\epsilon}\right) c_{2} b_{1}\right]+\left(a_{22} b_{1}-a_{12} b_{2}\right)\left[a_{12} c_{1} b_{2}-\left(a_{22}+\frac{1}{\epsilon}\right)\left(2 c_{1} b_{1}+c_{2} b_{2}\right)\right]}{\left.\left[a_{12} b_{2}\right) b_{2}-\left(a_{21} b_{1}+a_{22} b_{2}\right) b_{1}\right]\left(c_{1} b_{1}+c_{2} b_{2}\right),}
\end{aligned}
$$

the closed-loop transfer function is given by:

$$
-\frac{\left(c_{1} b_{1}+c_{2} b_{2}\right) s-\left(a_{22} c_{1} b_{1}+a_{11} c_{2} b_{2}-a_{12} c_{1} b_{2}-a_{21} c_{2} b_{1}\right)}{\left(c_{1} b_{1}+c_{2} b_{2}\right) s+\left(a_{22} c_{1} b_{1}+a_{11} c_{2} b_{2}-a_{12} c_{1} b_{2}-a_{21} c_{2} b_{1}\right)} \frac{1}{\epsilon s+1},
$$

\section{STATE FEEDBACK CONTROLLERS FOR SECOND-ORDER NONMINIMUM-PHASE NONLINEAR SYSTEMS}

In analogy with the linear case, in this section we will develop a class of nonlinear state feedback control laws for nonlinear nonminimum-phase systems, that result in a clused-loop response of a nonlinear first-order all-pass in series with a linear first-order lag. The development of the control law will be carried out in the normal form representation of the system, because it provides a convenient notational and conceptual framework. The basic result is stated in the following theorem:

Theorem 1 -Consider a second-order nonminimum-phase nonlinear system in its normal form (12):

$$
\begin{aligned}
& \zeta_{1}=F_{1}\left(\zeta_{1}, \zeta_{2}\right), \\
& \zeta_{2}=F_{2}\left(\zeta_{1}, \zeta_{2}\right)+G_{2}\left(\zeta_{1}, \zeta_{2}\right) u, \\
& y=\zeta_{2},
\end{aligned}
$$

$G_{2}\left(\zeta_{1}, \zeta_{2}\right) \neq 0$ and $\frac{\partial F_{1}}{\partial \zeta_{2}}\left(\zeta_{1}, \zeta_{2}\right) \neq 0$ 
Suppose that this system is subject to the state feedback:

$$
u=\frac{v-\psi\left(\zeta_{1}, \zeta_{2}\right)-\epsilon\left[\frac{\partial \psi}{\partial \zeta_{1}}\left(\zeta_{1}, \zeta_{2}\right) F_{1}\left(\zeta_{1}, \zeta_{2}\right)+\frac{\partial \psi}{\partial \zeta_{2}}\left(\zeta_{1}, \zeta_{2}\right) F_{2}\left(\zeta_{1}, \zeta_{2}\right)\right]}{\epsilon \frac{\partial \psi}{\partial \zeta_{2}}\left(\zeta_{1}, \zeta_{2}\right) G_{2}\left(\zeta_{1}, \zeta_{2}\right)},
$$

where $\psi\left(\zeta_{1}, \zeta_{2}\right)$ is a function that satisfies:

$$
\begin{aligned}
& \frac{\partial \psi}{\partial \zeta_{1}}\left(\zeta_{1}, \zeta_{2}\right)=-2 \frac{\frac{\partial F_{1}}{\partial \zeta_{1}}\left(\zeta_{1}, \zeta_{2}\right)}{\frac{\partial F_{1}}{\partial \zeta_{2}}\left(\zeta_{1}, \zeta_{2}\right)} \\
& \frac{\partial \psi}{\partial \zeta_{2}}\left(\zeta_{1}, \zeta_{2}\right)=-1
\end{aligned}
$$

on the line $F_{1}\left(\zeta_{1}, \zeta_{2}\right)=0$ and

$$
\frac{\partial \psi}{\partial \zeta_{2}}\left(\zeta_{1}, \zeta_{2}\right) \neq 0 \text { for every } \zeta_{1} \text { and } \zeta_{2} \text {. }
$$

Then, the resulting closed-loop system is equivalent, under appropriatc coordinate transformation, to a linear first-order lag in series with a nonlinear first-order all-pass.

Furthermore, the linear approximation of the closed-loop system around the steady state $\left(\zeta_{1 s}, \zeta_{2 s}\right)$ has the transfer function:

$$
-\frac{s-\frac{\partial F_{1}}{\partial \zeta_{1}}\left(\zeta_{1 s}, \zeta_{2 s}\right)}{s+\frac{\partial F_{1}}{\partial \zeta_{1}}\left(\zeta_{1 s}, \zeta_{2 s}\right)} \frac{1}{\epsilon s+1} .
$$

Proof-Under the control law (26), the closed-loop system is:

$$
\begin{aligned}
\zeta_{1}= & F_{1}\left(\zeta_{1}, \zeta_{2}\right), \\
\dot{\zeta}_{2}= & \frac{1}{\frac{\partial \psi}{\partial \zeta_{2}}\left(\zeta_{1}, \zeta_{2}\right)} \\
& \times\left[\frac{\partial \psi}{\partial \zeta_{1}}\left(\zeta_{1}, \zeta_{2}\right) F_{1}\left(\zeta_{1}, \zeta_{2}\right)+\frac{1}{\epsilon} \psi\left(\zeta_{1}, \zeta_{2}\right)-\frac{1}{\epsilon} v\right], \\
y= & \zeta_{2} .
\end{aligned}
$$

Under the coordinate change:

$$
\begin{aligned}
& \xi=\zeta_{1}, \\
& \eta=\psi\left(\zeta_{1}, \zeta_{2}\right),
\end{aligned}
$$

it becomes

$$
\begin{aligned}
\dot{\eta} & =-\frac{1}{\epsilon} \eta+\frac{1}{\epsilon} v, \\
\xi & =F_{1}[\xi, w(\xi, \eta)], \\
y & =w(\xi, \eta),
\end{aligned}
$$

where $\zeta_{2}=w(\xi, \eta)$ is the implicit function defined as the solution of $\eta=\psi\left(\xi, \zeta_{2}\right)$.
We have to verify that:

$$
\begin{aligned}
& \xi=F_{1}[\xi, w(\xi, \eta)], \\
& y=w(\xi, \eta),
\end{aligned}
$$

is a nonlinear first-order all-pass. At first, observe that from the definition of $w$ and the implicit function theorem:

$$
\begin{aligned}
& \frac{\partial w}{\partial \xi}(\xi, \eta)=-\frac{\frac{\partial \psi}{\partial \zeta_{1}}[\xi, w(\xi, \eta)]}{\frac{\partial \psi}{\partial \zeta_{2}}[\xi, w(\xi, \eta)]} \\
& \frac{\partial w}{\partial \eta}(\xi, \eta)=\frac{1}{\frac{\partial \psi}{\partial \zeta_{2}}[\xi, w(\xi, \eta)]}
\end{aligned}
$$

Therefore, the conditions (27) on $\psi$ translate into:

$$
\begin{aligned}
& \frac{\partial w}{\partial \xi}(\xi, \eta)--2 \frac{\frac{\partial F_{1}}{\partial \zeta_{1}}[\xi, w(\xi, \eta)]}{\frac{\partial F_{1}}{\partial \zeta_{2}}[\xi, w(\xi, \eta)]}, \\
& \frac{\partial w}{\partial \eta}(\xi, \eta)=-1,
\end{aligned}
$$

on the line $F_{1}[\xi, w(\xi, \eta)]=0$.

The above conditions make the system (32) a nonlinear first-order all-pass according to Proposition 3. Indecd, setting:

$$
\phi(\xi, \eta)=F_{1}[\xi, w(\xi, \eta)],
$$

one can verify Conditions $1,2^{\prime}, 3^{\prime}$ of Proposition 3 in a straightforward way. This completes the proof of the first part of the theorem. The derivation of the transfer function involves a straighforward linear approximation of the closed-loop system and use of the conditions on $\psi$.

Remark 6 -The condition $\partial \psi / \partial \zeta_{2}\left(\zeta_{1}, \zeta_{2}\right) \neq 0$ for every $\zeta_{1}, \zeta_{2}$, is necessary for the coordinate transformation (30) to be well-defined and invertible, over the entire $R^{2}$. Since $\partial \psi / \partial \zeta_{2}\left(\zeta_{1}, \zeta_{2}\right)=-1$ on the steady state line, there will always be a region around the operating steady state for which $\partial \psi / \partial \zeta_{2}\left(\zeta_{1}, \zeta_{2}\right) \neq 0$ will be satisfied; therefore the transformation (30) will be valid at least locally.

Remark 7-The state-feedback law (26) can be written in a more compact way using the Lie derivative notation:

$$
u=\frac{v-\psi-\epsilon \mathrm{L}_{F} \psi}{\epsilon \mathrm{L}_{G} \psi},
$$


where

$$
F=\left[\begin{array}{l}
F_{1}\left(\zeta_{1}, \zeta_{2}\right) \\
F_{2}\left(\zeta_{1}, \zeta_{2}\right)
\end{array}\right] \text { and } G=\left[\begin{array}{c}
0 \\
G_{2}\left(\zeta_{1}, \zeta_{2}\right)
\end{array}\right]
$$

Remark 8-The conditions (27) that $\psi$ has to satisfy do not determine it in a unique way (see also Remark 5). Consequently, (26) represents a class of control laws that guarantee stability of the closedloop system and provide a response of a nonlinear all-pass in series with a linear lag. However, depending on the choice of the function $\psi$, different performance characteristics of the closed-loop response will result.

Remark 9-The limiting closed-loop response as $\epsilon \rightarrow 0$ is easily found from (31) to be:

$$
\begin{aligned}
\zeta_{1} & =F_{1}\left[\zeta_{1}, w\left(\zeta_{1}, v\right)\right], \\
y & =w\left(\zeta_{1}, v\right) .
\end{aligned}
$$

In analogy with the linear case, we would expect that the above limiting response will be $I S E$-optimal for some $\psi$. We will see in the next section that this is indeed the case.

\section{ISE-OPTIMAL STATE FEEDBACK CONTROL OF NONLINEAR SYSTEMS}

In this section, we will provide a particular control law from the class of control laws (26), such that the resulting limiting closed-loop response (34) is $I S E$ optimal for step changes in the set point. Before we proceed in the calculation of the control law, consider the nonlinear system (12), initially at a reference steady state and let $v$ be a new constant set point value for the controlled variable $\zeta_{2}$. For this system, the ISE-optimization problem can be formulated as follows:

- Minimize:

$$
I S E=\frac{1}{2} \int_{0}^{\infty}\left[v-\zeta_{2}\right]^{2} \mathrm{~d} t
$$

subject to

$$
\begin{aligned}
& \zeta_{1}=F_{1}\left(\zeta_{1}, \zeta_{2}\right), \\
& \zeta_{2}=F_{2}\left(\zeta_{1}, \zeta_{2}\right)+G_{2}\left(\zeta_{1}, \zeta_{2}\right) u .
\end{aligned}
$$

Remark 10 -Notice that no constraints were posed in the formulation of the $I S E$-optimization problem. However, an obvious constraint that has to be satisfied is the internal stability of the closed-loop system.

The solution of the above optimization problem will specify the $I S E$-optimal response in the $\left(\zeta_{1}, \zeta_{2}\right)$ state space for a constant set point value $v$, and it will very naturally lead to calculation of the function $\psi$ which, when used in the control law (26), will result in this optimal response in the limit as $\epsilon \rightarrow 0$. The solution of the $I S E$-optimization problem involves a straightforward application of the necessary conditions for optimality. Following the standard procedure (for an outline see Johnson and Gibson, 1963), we find the following two singular ares in the $\left(\zeta_{1}, \zeta_{2}\right)$ state-space:

- Singular arc 1:

$$
\zeta_{2}=v
$$

- Singular arc 2:

$$
\zeta_{2}-2 \frac{F_{1}\left(\zeta_{1}, \zeta_{2}\right)}{\frac{\partial F_{1}}{\partial \zeta_{2}}\left(\zeta_{1}, \zeta_{2}\right)}=v
$$

The ISE-optimal trajectories will have to satisfy either (35) or (36). Since we are interested in ISEoptimality subject to internal stability, we have to check the internal stability characteristics of the system when the states are forced to satisfy either (35) or (36).

- Whenever the system is forced to follow Singular arc 1 (35), this corresponds to "perfect control"; the output $\zeta_{2}$ is identically equal to the set point $v$. The internal dynamics of the closed-loop system is then identical to the zero dynamics:

$$
\zeta_{1}=F_{1}\left(\zeta_{1}, v\right)
$$

and we already know that this will be stable for every $v$ if:

$$
\frac{\partial F_{1}}{\partial \zeta_{1}}\left(\zeta_{1}, \zeta_{2}\right)<0 \text { on the line } F\left(\zeta_{1}, \zeta_{2}\right)=0 \text {. }
$$

- Whenever the system is forced to follow Singular arc 2 (36), we do not have "perfect control" any more. The resulting closed-loop response will be given by:

$$
\begin{aligned}
\zeta_{1} & =F_{1}\left[\zeta_{1}, \Omega\left(\zeta_{1}, v\right)\right], \\
\mathrm{y} & =\Omega\left(\zeta_{1}, v\right),
\end{aligned}
$$

where $\zeta_{2}=\Omega\left(\zeta_{1}, v\right)$ is the implicit function defined as the solution of (36) for $\zeta_{2}$.

The stability characteristics of (37) will depend on the sign of:

$$
\frac{\partial F_{1}}{\partial \zeta_{1}}\left[\zeta_{1}, \Omega\left(\zeta_{1}, v\right)\right]+\frac{\partial F_{1}}{\partial \zeta_{2}}\left[\zeta_{1}, \Omega\left(\zeta_{1}, v\right)\right] \frac{\partial \Omega}{\partial \zeta_{1}}\left(\zeta_{1}, v\right),
$$

on the line $F_{1}\left[\zeta_{1}, \Omega\left(\zeta_{1}, v\right)\right]=0$.

Using the implicit function theorem and the definition of $\Omega$, we see that the above quantity becomes:

$$
-\frac{\partial F_{1}}{\partial \zeta_{1}}\left[\zeta_{1}, \Omega\left(\zeta_{1}, v\right)\right],
$$

on the line $F_{1}\left[\zeta_{1}, \Omega\left(\zeta_{1}, v\right)\right]=0$. Therefore, (37) will be stable for every $v$ if:

$$
\frac{\partial F_{1}}{\partial \zeta_{1}}\left(\zeta_{1}, \zeta_{2}\right)>0 \text { on the line } F\left(\zeta_{1}, \zeta_{2}\right)=0 \text {. }
$$

Based on the above arguments, it is obvious that following Singular are 1 will guarantee internal stability if and only if the system is minimum-phase, 
whereas following Singular arc 2 will guarantee internal stability if and only if the system is nonminimumphase. Therefore, for a nonminimum-phase system, forcing the closed-loop system to follow Singular arc 2 results in $I S E$-optimality and the $I S E$-optimal response is given by (37). Comparing the ISEoptimal response (37) with the limiting closed-loop response (34), we see that they become identical when $w=\Omega$, i.e. when:

$$
\psi\left(\zeta_{1}, \zeta_{2}\right)=\zeta_{2}-2 \frac{F_{1}\left(\zeta_{1}, \zeta_{2}\right)}{\frac{\partial F_{1}}{\partial \zeta_{2}}\left(\zeta_{1}, \zeta_{2}\right)}
$$

It is easy to show that the particular function $\psi$ given by (38) satisfies the conditions (27) of Theorem 1 . This leads to the following theorem:

Theorem 2-Consider a second-order nonminimum-phase nonlinear system in its normal form (12):

$$
\begin{aligned}
& \zeta_{1}=F_{1}\left(\zeta_{1}, \zeta_{2}\right), \\
& \zeta_{2}=F_{2}\left(\zeta_{1}, \zeta_{2}\right)+G_{2}\left(\zeta_{1}, \zeta_{2}\right) u, \\
& y=\zeta_{2},
\end{aligned}
$$

where $G_{2}\left(\zeta_{1}, \zeta_{2}\right) \neq 0$ and $\partial F_{1} / \partial \zeta_{1}\left(\zeta_{1}, \zeta_{2}\right) \neq 0$, which is subject to the state feedback:
The above theorem solves the ISE-optimal state feedback synthesis problem for second-order nonminimum-phase nonlinear systems in a way which is completely analogous to the linear case. The only inconvenience with the above theorem is that the result is expressed in terms of the normal form coordinates of the system. Transformation of the result in the original coordinates is not difficult and leads to:

Theorem 3-Consider a second-order nonminimum-phase nonlinear system of the form (8):

$$
\begin{aligned}
\dot{x}_{1} & =f_{1}\left(x_{1}, x_{2}\right)+g_{1}\left(x_{1}, x_{2}\right) u, \\
\dot{x}_{2} & =f_{2}\left(x_{1}, x_{2}\right)+g_{2}\left(x_{1}, x_{2}\right) u, \\
y & =h\left(x_{1}, x_{2}\right),
\end{aligned}
$$

where $\mathrm{L}_{g} h \neq 0$ and $\operatorname{det}\left[a d_{f}^{1} g \mid g\right] \neq 0$, which is subject to the state feedback:

$$
u=\frac{v-\psi\left(x_{1}, x_{2}\right)-\epsilon L_{f} \psi\left(x_{1}, x_{2}\right)}{\epsilon \mathbf{L}_{g} \psi\left(x_{1}, x_{2}\right)},
$$

where

$$
\psi\left(x_{1}, x_{2}\right)=h+2 \frac{\operatorname{det}[f \mid g]}{\operatorname{det}\left[a d_{j}^{1} g \mid g\right]} \mathbf{L}_{g} h .
$$

$$
u=\frac{v-\psi\left(\zeta_{1}, \zeta_{2}\right)-\epsilon\left[\frac{\partial \psi}{\partial \zeta_{1}}\left(\zeta_{1}, \zeta_{2}\right) F_{1}\left(\zeta_{1}, \zeta_{2}\right)+\frac{\partial \psi}{\partial \zeta_{2}}\left(\zeta_{1}, \zeta_{2}\right) F_{2}\left(\zeta_{1}, \zeta_{2}\right)\right]}{\epsilon \frac{\partial \psi}{\partial \zeta_{2}}\left(\zeta_{1}, \zeta_{2}\right) G_{2}\left(\zeta_{1}, \zeta_{2}\right)},
$$

where

$$
\psi\left(\zeta_{1}, \zeta_{2}\right)=\zeta_{2}-2 \frac{F_{1}\left(\zeta_{1}, \zeta_{2}\right)}{\frac{\partial F_{1}}{\partial \zeta_{2}}\left(\zeta_{1}, \zeta_{2}\right)}
$$

Assume that:

$$
\frac{\partial \psi}{\partial \zeta_{2}}\left(\zeta_{1}, \zeta_{2}\right) \neq 0 \text { for every } \zeta_{1} \text { and } \zeta_{2}
$$

Then, the resulting closed-loop response is equivalent, under appropriate coordinate transformation, to a linear first-order lag in series with a nonlinear first-order all-pass.

Furthermore, the closed-loop response is ISEoptimal for step changes in the set point $v$, in the limit as $\epsilon \rightarrow 0$.

Remark 11 -The condition $\partial \psi / \partial \zeta_{2}\left(\zeta_{1}, \zeta_{2}\right) \neq 0$ is necessary not only to make the control law (39) finite, but also for the solvability of (36) for $\zeta_{2}$.

Remark 12-Clearly, $\partial \psi / \partial \zeta_{2}\left(\zeta_{1}, \zeta_{2}\right)=-1$ on the steady state line. This implies that there will always exist a region around the desirable steady state for which $\partial \psi / \partial \zeta_{2}\left(\zeta_{1}, \zeta_{2}\right) \neq 0$. As long as the entire trajectory, including the initial conditions, lies within this region, the result of the theory will hold.
Assume that:

$$
\mathrm{L}_{g} \psi \neq 0 \text { for every } x_{1} \text { and } x_{2} \text {. }
$$

Then, the resulting closed-loop response is equivalent, under appropriate coordinate transformation, to a linear first-order lag in series with a nonlinear first-order all-pass.

Furthermore, the closed-loop response is ISEoptimal for step changes in the set point $v$, in the limit as $\epsilon \rightarrow 0$.

\section{EXAMPLE}

Consider a continuous stirred tank reactor, where the isothermal series/parallel Van de Vusse reaction (Van de Vusse, 1964; Kantor, 1986) is taking place:

$$
\begin{gathered}
A \rightarrow B \rightarrow C, \\
2 A \rightarrow D .
\end{gathered}
$$

The rates of formation of $A$ and $B$ are assumed to be:

$$
\begin{aligned}
& r_{\mathrm{A}}=-k_{1} c_{\mathrm{A}}-k_{3} c_{\mathrm{A}}^{2}, \\
& r_{\mathrm{B}}=k_{1} c_{\mathrm{A}}-k_{2} c_{\mathrm{B}},
\end{aligned}
$$

where $k_{1}=50 \mathrm{~h}^{-1}, k_{2}=100 \mathrm{~h}^{-1}, k_{3}=10 \mathrm{l}(\mathrm{gmol} \cdot \mathrm{h})^{-1}$ are the reaction rate constants. The feed stream 
consists of pure $A$. The mass balances for $A$ and $B$ are given by:

$$
\begin{gathered}
V \frac{\mathrm{d} c_{\mathrm{A}}}{\mathrm{d} t}=F\left(c_{\mathrm{A}_{0}}-c_{\mathrm{A}}\right)+V\left(-k_{1} c_{\mathrm{A}}-k_{3} c_{\mathrm{A}}^{2}\right), \\
V \frac{\mathrm{d} c_{\mathrm{B}}}{\mathrm{d} t}=F\left(-c_{\mathrm{B}}\right)+V\left(k_{1} c_{\mathrm{A}}-k_{2} c_{\mathrm{B}}\right),
\end{gathered}
$$

where $F$ is the inlet flowrate of $A, V$ is the reactor volume which is kept constant during the operation, $c_{\mathrm{A}}$ and $c_{\mathrm{B}}$ are the concentrations of the species $A$ and $B$ inside the reactor, respectively, and $c_{\mathrm{A}_{0}}=$ $10 \mathrm{gmol} \cdot \mathrm{l}^{-1}$ is the concentration of $A$ in the feed stream. We wish to maintain $c_{B}$ at its set point, by manipulating the dilution rate $F / V$. Initially the system is at steady state with $c_{A_{r}}=3 \mathrm{gmol} \cdot 1^{-1}$ and $c_{B_{r}}=1.117 \mathrm{gmol} \cdot 1^{-1}$. By letting:

$$
\begin{aligned}
x_{1} & =c_{\mathrm{A}}, \\
x_{2} & =c_{\mathrm{B}}, \\
u & =\frac{F}{V}, \\
y & =c_{\mathrm{B}},
\end{aligned}
$$

the system is put in the standard form (8):

$$
\begin{aligned}
& \dot{x}_{1}=-k_{1} x_{1}-k_{3} x_{1}^{2}+\left(c_{\mathrm{A}_{0}}-x_{1}\right) u, \\
& \dot{x}_{2}=k_{1} x_{1}-k_{2} x_{2}-x_{2} u, \\
& y=x_{2} .
\end{aligned}
$$

One can easily verify that the system satisfies condition (9):

$$
\begin{array}{r}
g_{1}\left(x_{1}, x_{2}\right) \frac{\partial h}{\partial x_{1}}\left(x_{1}, x_{2}\right)+g_{2}\left(x_{1}, x_{2}\right) \frac{\partial h}{\partial x_{2}}\left(x_{1}, x_{2}\right) \\
=-x_{2} \neq 0 .
\end{array}
$$

A nontrivial particular solution of the partial differential equation (10) is $t(x)=\left(c_{\mathrm{A}_{0}}-x_{1}\right) / x_{2}$. Thus, the change of coordinates:

$$
\zeta=\left[\begin{array}{l}
t(x) \\
h(x)
\end{array}\right]=\left[\begin{array}{c}
\frac{c_{\mathrm{A}_{0}}-x_{1}}{x_{2}} \\
x_{2}
\end{array}\right],
$$

transforms (43) in its Byrnes-Isidori normal form:

$$
\begin{aligned}
& \zeta_{1}=F_{1}\left(\zeta_{1}, \zeta_{2}\right), \\
& \zeta_{2}=F_{2}\left(\zeta_{1}, \zeta_{2}\right)+G_{2}\left(\zeta_{1}, \zeta_{2}\right) u, \\
& y=\zeta_{2},
\end{aligned}
$$

where

$$
\begin{aligned}
& F_{1}\left(\zeta_{1}, \zeta_{2}\right) \\
& =\frac{k_{1}\left(1-\zeta_{1}\right)\left(c_{\mathrm{A}_{0}}-\zeta_{1} \zeta_{2}\right)+k_{2} \zeta_{1} \zeta_{2}+k_{3}\left(c_{\mathrm{A}_{0}}-\zeta_{1} \zeta_{2}\right)^{2}}{\zeta_{2}} \\
& \quad F_{2}\left(\zeta_{1}, \zeta_{2}\right)=k_{1}\left(c_{\mathrm{A}_{0}}-\zeta_{1} \zeta_{2}\right)-k_{2} \zeta_{2} \\
& \quad G_{2}\left(\zeta_{1}, \zeta_{2}\right)=-\zeta_{2}
\end{aligned}
$$

The zero dynamics of (46) is $\dot{z}=F_{1}(z, y)$, i.e.

$$
\dot{z}=\frac{k_{1}(1-z)\left(c_{\mathrm{A}_{0}}-z y\right)+k_{2} z y+k_{3}\left(c_{\mathrm{A}_{\mathrm{V}}}-z y\right)^{2}}{y},
$$

where $y$ assumes a constant value.

Based on Proposition 2, the sign of:

$\frac{\partial F_{1}}{\partial z}(z, y)=\frac{k_{1}\left(2 z y-y-c_{\mathrm{A}_{0}}\right)+k_{2} y-2 k_{3} y\left(c_{\mathrm{A}_{0}}-z y\right)}{y}$,

or, in the original coordinate system $\left(x_{1}, x_{2}\right)$, the sign of:

$$
k_{2}-k_{1}-2 k_{3} x_{1}+k_{1} \frac{c_{A_{0}}-2 x_{1}}{x_{2}},
$$

locally around the steady state, will determine whether the system is minimum- or nonminimumphase. For the initial steady state $\left(x_{1 s}=3.0\right.$, $\left.x_{2 s}=1.117\right)$ and the values of the reaction rate constants, one can easily check that the quantity (52) is positive and therefore the operating steady state lies in the nonminimum-phase region of the steady state line. The open-loop behavior of both the nonlinear system and the linearized around the initial steady state system is shown in Fig. 1, for a negative step change at the input from $u=34.28$ to 25 at time $=0$. Clearly, in a neighborhood around the initial steady state, the nonlinear response is successfully approximated by the linearized one. An inverse response can be observed in both cases, as a result of the unstable zero dynamics (or the positive zero in the linearized system). The nonlinear state-feedback control law is obtained from (40):

$$
u=\frac{v-\psi\left(x_{1}, x_{2}\right)-\epsilon \mathrm{L}_{f} \psi\left(x_{1}, x_{2}\right)}{\epsilon \mathrm{L}_{g} \psi\left(x_{1}, x_{2}\right)}
$$

where

$$
\begin{gathered}
\psi\left(x_{1}, x_{2}\right)=h+2 \frac{\operatorname{det}[f \mid g]}{\operatorname{det}\left[\operatorname{ad}_{f}^{1} g \mid g\right]} L_{g} h, \\
f=\left[\begin{array}{c}
-k_{1} x_{1}-k_{3} x_{1}^{2} \\
k_{1} x_{1}-k_{2} x_{2}
\end{array}\right], \quad g=\left[\begin{array}{c}
c_{A_{0}}-x_{1} \\
-x_{2}
\end{array}\right], \quad h=x_{2} .
\end{gathered}
$$

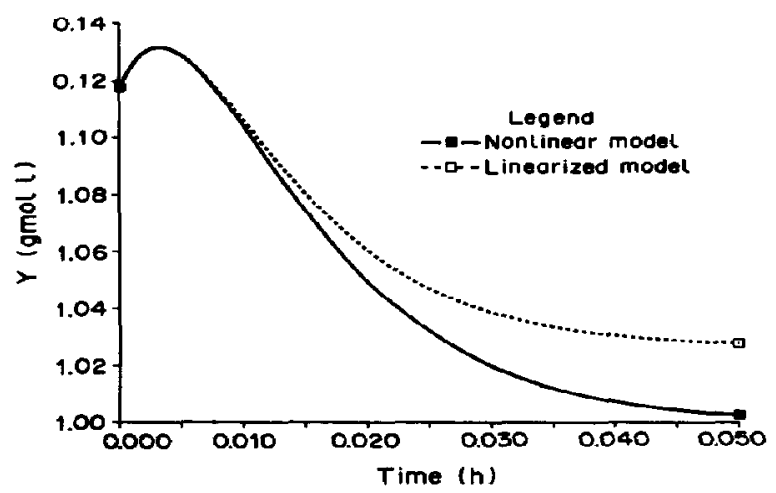

Fig. 1. Open-loop response. 


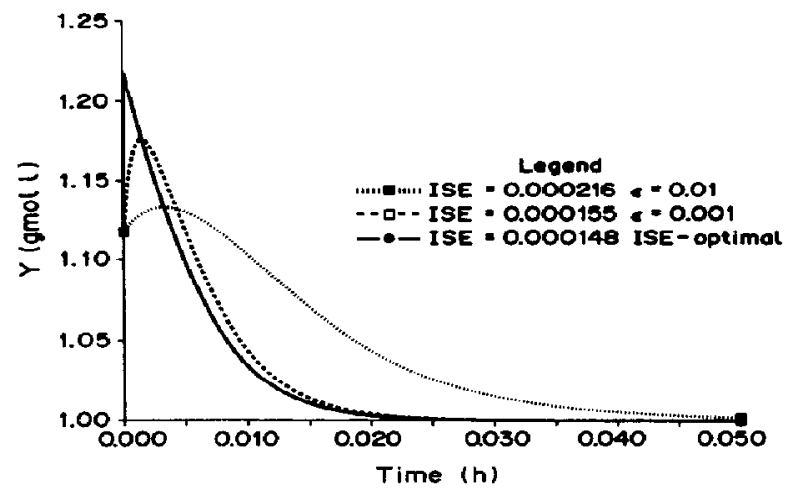

Fig. 2. Closed-loop response.

The response of the closed-loop system for a negative set point change in the output $y$ from 1.117 to 1 is shown in Fig. 2, for different values of the adjustable parameter $\epsilon$. Decreasing values of $\epsilon$ result in a faster closed-loop response and smaller $I S E$ values (at the cxpense of larger input values of course). As $t \rightarrow 0$, the $I S E$-optimal response (37) is obtained.

Figure 3 provides the local picture of the Singular arc 2 (36) together with the steady state line, in the region of interest in the $\left(\zeta_{1}, \zeta_{2}\right)$ state space. Notice that the particular singular arc corresponds to the specific postulated final set point, $v=1$. Under the control law (40), the closed-loop trajectory starts from the initial steady state $\left(\zeta_{1}-6.26, \zeta_{2}=1.117\right)$, instantaneously jumps to the singular arc at $\left(\zeta_{1}=6.26, \zeta_{2}=1.22\right)$, and then evolves along the singular arc until it reaches the final steady state $\left(\zeta_{1}=7.5, \zeta_{2}=1\right)$.

Remark 13-Because of the shape of the singular arc, the trajectory of the closed-loop system for the specified final steady state, depends drastically on the initial steady state. For example, in the case that the initial steady state lies in the region which is to the right of the singular arc (i.e. $\zeta_{1}>9$ ), the condition that guarantees solvability of $(36)$ for $\zeta_{2}$ and consequently the result of Theorems 2 and 3 do not hold. Such pathological cases may occur and the present work does not address them.

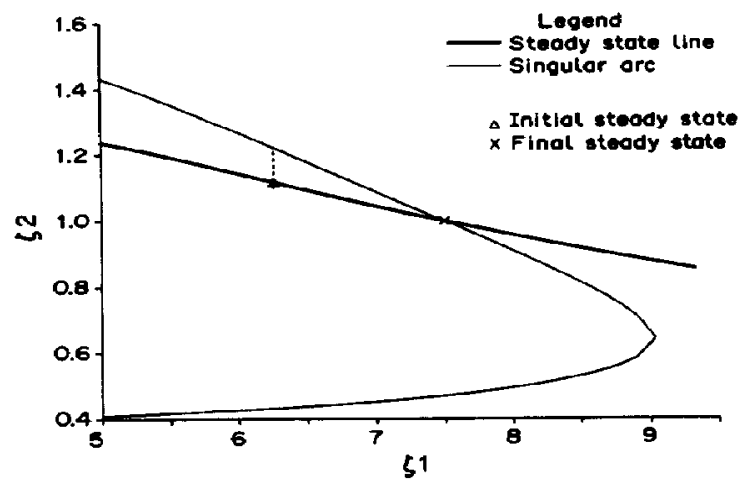

Fig. 3. Singular arc and steady state line.

\section{CONCLUSIONS}

The present work addressed the problem of nonlinear state feedback control of second-order nonminimum-phase systems. A class of control laws was developed that results in a closed-loop response of a nonlinear first-order all-pass in series with a linear first-order lag. A particular choice of the control law leads to $I S E$-optimal closed-loop response. The proposed methodology is analogous to the one of placing poles at the "mirror image" of right-half-plane zeros in linear systems. The performance of the control law in set point tracking was successfully tested, through simulations in a CSTR example.

Acknowledgement-The authors are indebted to Coleman Brosilow, who suggested the idea of imposing a closed-loop response with poles at the reflection of right-half plane zeros at each point of linearization. His comments and insights were invaluable.

\section{NOMENCLATURE}

$C_{i}=$ Concentration of species $i$ in the reactor (g mol 1-1).

$F=$ Volumetric feedrate $\left(h^{-1}\right)$

$V=$ Reactor volume (1)

$k_{1}, k_{2}=$ Rate constants for first-order reaction $\left(\mathrm{h}^{-1}\right)$

$k_{3}=$ Rate constant for second-order reaction, (1 g IIIOl h $\mathbf{h}^{-1}$ )

$R^{2}=$ Two-dimensional Euclidian space

$u=$ Manipulated input

$v=$ Set point

$x=$ Vector of state variables

$y=$ Output

$f, g=$ Vector fields

$f_{1}, f_{2}=$ Components of the vector field $f$

$g_{1}, g_{2}=$ Components of the vector field $g$

$h, t=$ Scalar fields

$F_{1}, F_{2}=$ Scalar fields in the normal form

$G_{2}=$ Scalar field in the normal form

$s=$ The Laplace domain variable

Greek letters

$\zeta=$ Transformed state variables

$\epsilon=$ Adjustable parameter in the control law

$\psi=$ Scalar function appearing in the control law

$\mathbf{\Omega}=$ Scalar function that corresponds to the ISEoptimal closed-loop response

Math symbols

$$
\begin{aligned}
a d_{f}^{1} g= & \frac{\partial g}{\partial x} f-\frac{\partial f}{\partial x} g \text { (first-order Lie bracket between the } \\
& \text { vector fields } f \text { and } g \text { ) } \\
\operatorname{det}[-]= & \text { Determinunt of a mutrix } \\
\mathrm{L}_{g} h= & \frac{\partial h}{\partial x_{1}} g_{1}+\frac{\partial h}{\partial x_{2}} g_{2} \text { (Lie derivative of the scalar field } h \\
& \text { with respect to the vector field } g \text { ) }
\end{aligned}
$$

\section{REFERENCES}

Byrnes C. I. and A. Isidori, Global feedback stabilization of nonlinear systems. Proc. 24th IEEE CDC, Ft Lauderdale, p. 1031 (1985).

Economou C. G., M. Morari and B. O. Palsson, Internal model control. 5. Extension to nonlinear systems. Ind. Engng Chem. Process Des. Dev. 25, 403 (1986). 
Garcia C. E. and M. Morari, Internal model control. 1. $A$ unifying review and some new results. Ind. Engng Chem. Process Des. Dev. 21, 308 (1982).

Isidori A. and C. H. Moog, On the equivalent of the notion of transmission zeros. Modeling and Adaptive Control (C. I. Byrnes and A. H. Kurszhanski, Eds). Springer, New York (1988).

Johnson C. D. and J. E. Gibson, Singular solutions in problems of optimal control. IEEE Trans. Automat. Control AC-8, 4 (1963).

Kantor J. C., Stability of state feedback transformations for nonlinear systems-some practical considerations. Proc. 1986 ACC, Seattle, p. 1014 (1986).

Kravaris C., Input/output linearization: a nonlinear analog of placing poles at the process zeros. AIChE $J l 34,1803$ (1988).

Kravaris C. and C. B. Chung, Nonlinear state feedback synthesis by global inpul/output linearization. AIChE $J l$ 33, 592 (1987).

Parrish J. R. and C. B. Brosilow, Nonlinear inferential control. AIChE Jl 34, 633 (1988).

Van de Vusse J. G., Chem. Engng Sci. 19, 994 (1964). 